\title{
The Error of our Ways: The experience of Self- Reported Position in a Location-Based Game
}

\author{
Steve Benford ${ }^{1}$ Will Seager $^{1}$, Martin Flintham ${ }^{1}$, Rob Anastasi ${ }^{1}$, Duncan Rowland ${ }^{1}$, Jan \\ Humble $^{1}$, Danaë Stanton ${ }^{1}$, John Bowers ${ }^{1}$, Nick Tandavanitj ${ }^{2}$, Matt Adams ${ }^{2}$, Ju Row \\ Farr $^{2}$, Amanda Oldroyd ${ }^{3}$, Jon Sutton ${ }^{3}$ \\ ${ }^{1}$ Mixed Reality Laboratory, The University of Nottingham, Nottingham, NG8 1BB, UK \\ \{sdb, wps, mdf, rma, dar, jch, des\}@cs.nott.ac.uk \\ ${ }^{2}$ Blast Theory, Unit 43a, Regent Studios, 8 Andrews Road, London, E8 4QN, UK \\ \{nick, matt, ju\}@blasttheory.co.uk \\ ${ }^{3}$ BT Exact, Ross Building PP4, Adastral Park, Martlesham, Ipswich IP5 3RE, UK \\ \{amanda.oldroyd, jon. sutton\}@bt. com
}

\begin{abstract}
We present a study of people's use of positional information as part of a collaborative location-based game. The game exploits self-reported positioning in which mobile players manually reveal their positions to remote players by manipulating electronic maps. Analysis of players' movements, position reports and communications, drawing on video data, system logs and player feedback, highlights some of the ways in which humans generate, communicate and interpret position reports. It appears that remote participants are largely untroubled by the relatively high positional error associated with self reports. Our analysis suggests that this may because mobile players declare themselves to be in plausible locations such as at common landmarks, ahead of themselves on their current trajectory (stating their intent) or behind themselves (confirming previously visited locations). These observations raise new requirements for the future development of automated positioning systems and also suggest that selfreported positioning may be a useful fallback when automated systems are unavailable or too unreliable.
\end{abstract}

\section{Introduction}

In recent years there has been a proliferation of interest in systems which exploit positional information to support mobile interactivity. The Xerox PARCTab [14] and Olivetti's Active Badge system [13] provide early examples which have inspired increasing interest in the design of location-aware mobile applications. For many researchers, obtaining reliable positional information for users or devices is seen as an essential aspect of delivering context aware services. For example, Cyberguide [1] employs indoor and outdoor positioning technologies to create a mobile tour guide. Context aware, position-informed approaches have also been proposed in domains as varied as information retrieval [5], workplace activity tracking [9] and network routing and resource discovery [7]. 
However, there have been relatively few reports of large-scale deployments of these kinds of location-based applications and we do not yet have a detailed understanding how end users actually use and interpret positional data. The few reports that have been published raise significant challenges for the design of interfaces, applications and the underlying positioning systems. For example, our own previous studies of a location-based artistic game called 'Can You See Me Now?' as it toured several cities, involving several hundred players in each over a period of several days, yielded rich and detailed accounts of how people experienced GPS as a positioning technology $[3,6]$. 'Can You See Me Now?' was a game of tag in which online players, logged on to the game over the Internet, were chased through a virtual model of a city by street players who, equipped with handheld computers, wireless networking and GPS receivers, had to run through the actual city streets in order to catch them. Online players could also 'tune in' to a real-time audio stream from the street players and could send them text messages in return.

Analysis of the communication between and movements of street and online players revealed that the performance of GPS has a major impact on the game. This stemmed from both the error associated with GPS measurements but - significantly also its availability; it was often difficult to obtain a good enough GPS fix while running around the city to be able to play the game. Online players experienced these problems in various ways: they were sometimes unaware of them, but at other times they were revealed in a jarring way; and occasionally the players even interpreted them as part of the game or exploited them tactically. Street players on the other hand, were constantly aware of GPS performance. For them, the experience was as much an ongoing battle to obtain a reliable GPS fix as it was about chasing online players. This is not to say that GPS is a poor technology - but rather, that it cannot just be deployed on the streets of a real city and be expected to work continually and seamlessly over the course of several days. Rather than the technology being invisible, street players had to learn to make it work for them, gradually building up a stock of knowledge of its behaviour at particular locations and times.

This paper builds on this previous experience through a study of a further touring artistic game called 'Uncle Roy All Around You'. This has used an alternative 'lowtech' positioning system called self reported positioning in which mobile players declare their own positions to the game server, both explicitly and implicitly, through their use of an electronic map. There are two motivations behind this study.

First, we wish to deepen our understanding of the human issues involved in using positioning systems. The use of self-reported positioning in 'Uncle Roy All Around You' provides a useful vehicle for exploring how end-users collaboratively generate and interpret positional data for themselves as part of a large-scale publicly deployed application. By analysing human behaviour we are able to uncover broader implications for automated positioning systems and beyond this, for the way in which we approach positioning in general. Furthermore, experience with low-tech self-reported positioning can be seen as establishing a baseline of experience against which automated positioning technologies might subsequently be compared.

Second, we are interested in the technique of self-reported positioning in its own right, i.e., as an alternative to, or safety net for, automated positioning systems in situations where they might be unavailable or too unreliable - for example, where 
there isn't sufficient coverage across an urban environment or where they will be used by users who are unfamiliar with their characteristics.

\section{Method}

As with our previous study of 'Can You See Me Now?', our method involves a naturalistic study of a professional-quality application that is publicly deployed and experienced in a realistic setting - the streets of a city - by a large number of people hundreds of participants - over many days. Our study draws on three sources of data: video-based ethnographic observations of selected participants; direct feedback from participants through questionnaires and subsequent emails and face-to-face discussions; and system logs of all participants' movements and communications. Between them, these sources enable us to build a rich picture of the experience. This approach builds upon a rich tradition of using ethnography to inform system design by studying the use of technologies 'in the wild', i.e., situated in the real-world rather in an artificially controlled settings such as a laboratory, where they are subject to all of the contingencies that this introduces.

Our chosen application is again an artistic game; a touring interactive performance that has been produced in collaboration with professional artists. Our game focuses on collaboration between mobile street players and remote online players and in particular on how the latter can guide the former on a journey through the city. We have chosen this application for two reasons. First, games and artworks provide a good vehicle for engaging the public in large scale experiments. They are engaging, can be deployed in public, can mimic a variety of situations and behaviours; and yet are safe - they involve minimal risk when compared to deploying say, a safety critical system. Second, we anticipate that games will emerge as a major market for ubiquitous technologies, in the same way that conventional games have been a major driving force behind the development of computing technologies, even if this has not always been recognized by the research community. Indeed, several research projects have begun to explore the challenges involved in delivering games on the streets including Pirates! [4], AR Quake [12], Mindwarping [11] and 'Can You See Me Now?' [3].

Positioning systems are an essential but also problematic aspect of such games. Although a variety of systems is available including GPS, cellular positioning, radio pingers, video tracking, inertial systems and others, these vary greatly in terms of cost, availability, coverage, resolution, frequency and accuracy. In particular, there is currently no universal tracking system that can provide reliable, accurate and extensive coverage across a city with the result that game developers and players have to cope with considerable uncertainty with regard to location. 


\section{Self-reported positioning}

With self-reported positioning mobile players declare their own positions to the game rather than having them determined by an automated positioning system such as GPS. Our proposal for self-reported positioning actually consists of two related mechanisms that determine position in different ways. In the first, players explicitly declare their position to the game server by interacting with an electronic map, in effect saying 'I am here', in return for location relevant game content such as clues or messages from other players.

In the second, players interact with the electronic map in the natural course of wayfinding. However, their interface, which is delivered on a handheld computer, only allows them to see a limited area of the overall game map at any moment in time, requiring them to pan and zoom their viewpoint. Their current view of the map (a rectangular area) is then taken as an indication of their likely position within the physical world. In short, we assume that where they are looking on the map indicates where they actually are. This second mechanism can be described as implicitly selfreported position as it may be transparent to the player who could be unaware that their map manipulations are being interpreted as positions.

This approach is certainly low-cost and also has high availability when compared to systems such as GPS. On the other hand, there is no guarantee that it will produce accurate positional information. Players might be mistaken about where they are or might choose to deliberately lie about their location, and it is far from clear that where you are looking on a map is necessarily a reliable indication of where you are. We have therefore undertaken a study in which we piloted this approach as part of a location-based game that was experienced by members of the public.

\section{An overview of Uncle Roy All Around You}

'Uncle Roy All Around You' is a location-based game that mixes street players who journey through a city in search of an elusive character called Uncle Roy, in interaction with online players who journey through a parallel 3D model of the city, who are able to track the street players, communicate with them and can choose to help or hinder them. The aim of the game is to create an engaging collaborative experience for street and online players based around the theme of trust in strangers.

On arrival at the venue a street player is given a handheld computer, is briefed that their mission is to rendezvous with Uncle Roy and is shown how to use the interface. On entering the city, their first task is to find a red marker on the map, to get to the physical location that this indicates, and then declare their position to Uncle Roy. Once they have achieved this, they move on to the second phase of the game in which 'Uncle Roy' (the game) sends them a series of clues in response to further declarations of position. These clues lead them through the park and into the narrow city streets in search of Uncle Roy's office. During this time, the street player may also receive text messages from online players who are following their progress and who may offer them advice, directions or otherwise. In return, the street player is able to record and 
upload short (seven second) audio messages for the online players to hear. Eventually they find their way to a physical office and the game switches into its final phase, the details of which are beyond the scope of the present paper.
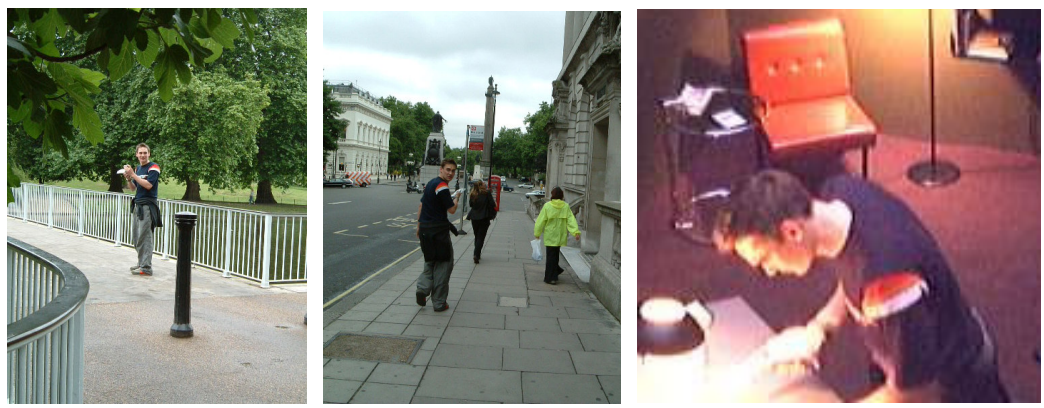

Figure 1: street player's experience: the park, streets and office

An online player connected to the game over the Internet journeys through a parallel 3D model of the game space. They move their avatar through this model using the arrow keys on their keyboard, encounter other online players and can send them text messages. They also access a set of on-screen cards that provide details of the current street players, see representations of these players' positions in the model, and can exchange text and audio messages with them as described above. Online players can find additional information in the 3D model, including the location of Uncle Roy's office, which they can use to guide the street players. Finally, online players can 'join' street players in Uncle Roy's office via a live webcam in the final phase of the game.

'Uncle Roy All Around You' was piloted in central London over two weeks in May and June of 2003. During this time it was experienced by 272 street players and over 440 online players. A strong positive reaction from players (through questionnaires and email feedback) and press suggests that we created an engaging experience. However, the overall success of the experience is not our concern in this paper. Instead, we are interested in its use of self-reported position.

\section{Implementing self-reported positioning}

The street player's interface to 'Uncle Roy All Around You' takes the form of the interactive map shown in figure 2. The overall size of the game map is 1600 by 1000 meters. The street player views this through a 280 by 320 pixel view area and can swap between two zoom settings: zoomed out, in which one pixel is equivalent to four meters, giving a viewable area of 1120 by 1280 square meters (most of the map); and zoomed in, in which one pixel is equivalent to one meter, giving a viewable area of 280 by 320 square meters. The street player can also rotate the map. 

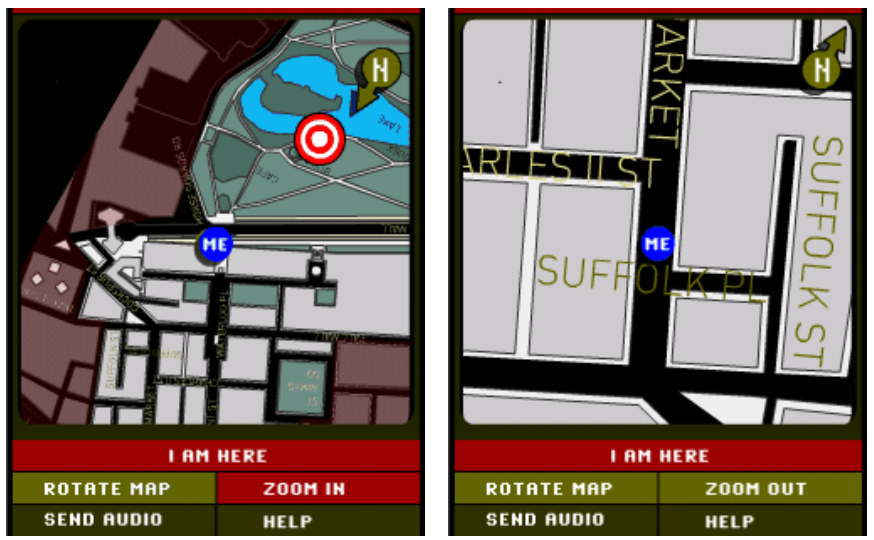

Figure 2: street player's map, zoomed out and in

The player pans their view over the map by using a stylus to drag the 'me' icon (a circle of radius of 10 pixels labeled with the word 'me') to a new position. The map then re-centers itself around this position. It is possible to place this icon anywhere on the map, including inside buildings and in the lake, and also to move off of the visible edge of the map in which case the display appears blank. This approach to navigating the map was chosen over other approaches such as using sliders, scrollbars, buttons and thumbwheels, because it allows simultaneous panning in two dimensions with just one simple manipulation, and also because it implies a relationship between the map view and the player's physical location.

Implicit position updates (giving $\mathrm{x}$ and $\mathrm{y}$ coordinates and rotation and zoom settings) are sent to the game server whenever the player pans, zooms or rotates their view of the map. We refer to these implicit position updates as 'map manipulations'. In order to explicitly declare their position, the player positions the 'me' icon at the appropriate place and then presses the 'I AM HERE' button, sending a 'declaration' event to the game server.

The street player receives a different text clue back from the game server depending on which of 49 regions they declare themselves to be in. These regions vary in size from roughly 150 by 150 meters in the open park area down to roughly 10 by 10 meters in the narrow city streets. A second successive declaration in a region returns a further clue. These clues and also messages from online players pop up over the map and need to be dismissed before further interaction is possible.

Two outer regions bound the game zone and return clues that are intended to guide the player back towards the middle of the map. The innermost of these returns the message: "The policeman was firm but polite, not this way today" followed by (on a second declaration) "You are off track"; while the larger outmost region returns the message "I cannot guide you out here. You have got lost. Go back the way you came" followed by "Retrace your steps, you are too far away and in the wrong place".

The online players interface is shown in figures 3 and 4 . The white avatar represents this player, the cards on the right show the current street players and the text boxes at the bottom are for sending text messages to online players or individual street 
players. Online players can also switch between a first person and bird's-eye view of the model. They see different representations of map manipulations and declarations. The former are represented by the position of a pulsing red sphere, which is labelled with the street player's name (figure 3).

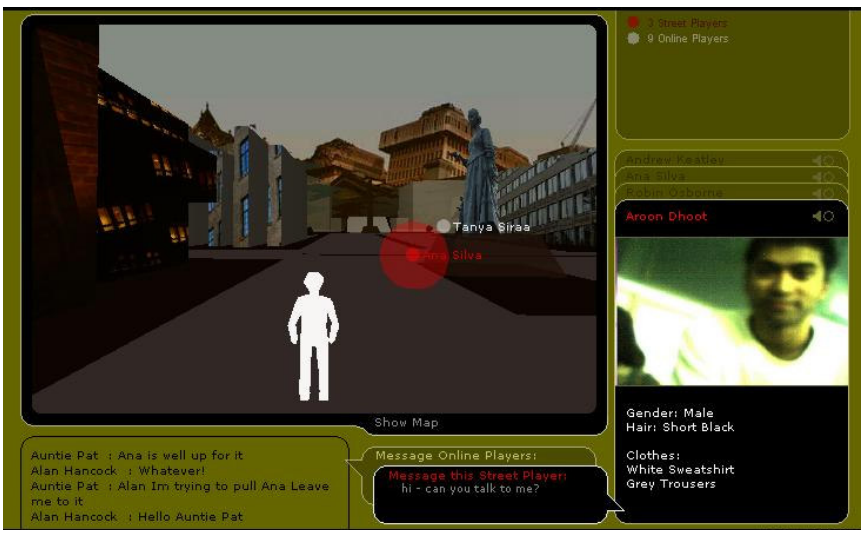

Figure 3: an online player observes a map manipulation

In contrast, declarations are portrayed in a far more dramatic and eye catching manner: over the course of a few seconds a dramatic sound is played, radiating lines emanate from the red sphere, while a much larger translucent sphere appears in the 3D model and gradually shrinks (like a deflating balloon) down to the street player's newly declared position (figure 4). These effects are intended to make declarations highly noticeable and in the case of the shrinking sphere, to give some sense of the street player's location in the 3D model, even when seen from some distance way.

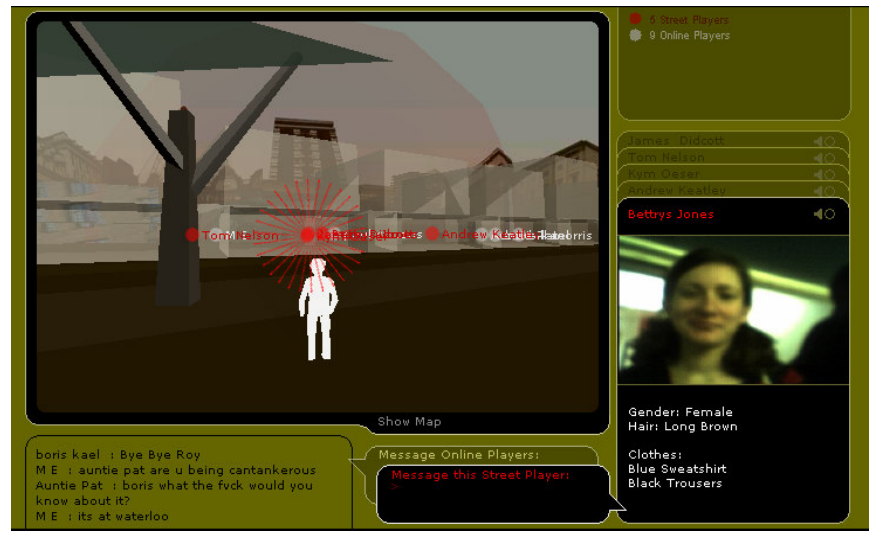

Figure 4: an online player observes a declaration 


\section{Performance of self-reported positioning}

Our analysis of the use of self-reported positioning draws on three sources of data: system logs of all declarations, map manipulations and text and audio messages from players; feedback from street and online players via email and questionnaires; and video observation of some players.

Our first (rather obvious) observation is that self-reported positioning provided excellent coverage and availability. Street players quickly learned to use it; it was not necessary to wait to get a fix on sensors or satellites; and there were no black spots within the game zone (there were wireless communications problems however, which made it impossible to transmit position updates, although these would have equally affected an on-board positioning system such as GPS). The equally obvious downside is that players had to work to generate position updates themselves (at least the explicit declarations) so that the positioning technology was not invisible. We return to this point later on in the conclusions.

This said, we now continue our analysis by treating self-reported positioning as if it were a technology whose performance (in a narrow technical sense) needs be measured, as this is typical of the way in which automated positioning systems such as GPS are discussed and compared. We focus on three key characteristics of performance: frequency, resolution and accuracy.

To determine frequency and resolution we have analyzed system logs of the 5,309 declarations and 18,610 map manipulations that were generated by all 272 street players. The distributions of duration, distance moved and errors that are discussed below are skewed, with some high outlying values, and so it is most informative to summarize them using the median, and the inter-quartile range. The median duration of declaration events (time between successive declarations by the same player) is 1.14 minutes (inter-quartile range of $1.31=1.98-0.67$ ) whereas the median duration of map manipulations is 0.11 minutes (inter-quartile range of $0.65=0.68-0.03$ ). In other words, declarations occur approximately once every minute whereas map manipulations are roughly ten times more frequent.

The maximum possible spatial resolution of position updates was 1 meter $(1$ screen pixel equates to 1 meter on the map when zoomed in). However, in practice, updates fall further apart than this. The median distance moved across the map between successive declarations by the same player was 80 meters (inter-quartile range of $82=135$ 43 ) and between map manipulations was 40 meters (inter-quartile range of $88=90-2$ ).

Analyzing accuracy involves comparing street players' self-reported positions with their actual positions in the physical world. We followed 10 players and recorded their progress on video. We then manually analyzed the video to transcribe their 174 declarations and 481 map manipulations, estimating the players' actual positions at the times when these events were generated (we believe that our estimates are accurate to within approximately five meters). We derive two measurements of accuracy from these observations. The first is 'distance error', the straight-line distance between reported and observed positions. The median distance error for declarations was 25 meters (inter-quartile range of $36=48-12$ ) and for map manipulations was 39 meters (inter-quartile range of $76=97-21$ ). However, there were a few position updates that 
were associated with particularly large errors. The maximum distance error for declarations was 240 meters and for map manipulations was 553 meters.

Our second way of expressing accuracy is in terms of 'off map' errors. These are declarations or map manipulations where the error is sufficiently large (greater than 120 pixels East-West or 160 pixels North-South) that the street player's actual physical position would not even appear on their view of the map. This reflects the idea that it is your entire map view, rather than the position of the central 'me' icon, that expresses where you are. 1.7\% (3 out of 174) of observed declarations were 'off map', compared to $8.3 \%$ (40 out of 481 ) of map manipulations.

In contrast to these figures, GPS typically produces a reading every second, has a resolution of a meter or so and depending on which kind of GPS is used (e.g., differential or not) and on local conditions, has a typical accuracy of between approximately one and ten meters. For example, two previous experiences of using GPS as part of location-based games in similarly built up cities reported average errors (estimated by the GPS receivers themselves) of 4 meters (for differential GPS) and 12 meters (for non-differential), although, as with self reported positioning, there were occasionally very large errors (106 and 384 meters respectively), most likely due to multi-path reflections at specific locations [3].

At first sight, it seems that self-reported positioning produces less frequent, coarser and less accurate positional information than GPS and we might be tempted to conclude that it performs less well. However, two issues need to be borne in mind. First is availability. Reports of previous experiences noted that even GPS knowledgeable players had to work hard to obtain any GPS readings at all, exploiting knowledge of good GPS locations that they had built up over several days play, and even then they often could not obtain a GPS fix [3,6]. A driving motivation behind self-reported positioning was a concern that poor availability would make GPS too unreliable, especially in the hands of GPS 'naïve' players. Second, is the underlying nature of the 'errors' involved and their impact on the players, an issue that we now explore in detail by analyzing street and online players' experience of self-reported positioning.

\section{How online players use position reports}

In order to understand how self reported positioning is used in the game, we have examined the way in which online players used position information as part of their collaboration with street players. Specifically, we have analyzed the private text messages that they sent to street players to see to what extent they were confident in their knowledge of street player's positions or alternatively, whether they perceived reported positions as suspect or problematic. Of the 3,109 private text messages that were logged, approximately 1,670 were concerned with location in some way (the remainder being concerned with other aspects of social interaction). We coded these location oriented messages into five categories. The first category is messages in which the online player appears to have a precise enough fix on a street player's location to be able to give directions or tell the street player where they are. There are 735 such messages, such as:

The big street in front of you 
You are very close now

And stay on that side of the road

Literally meters away from you

U r very close step back 5 feet

Stop take a right NOW

It is notable how readily and commonly 'deictical' linguistic elements (in front, close, right, left, there, here - terms which have a sense when one knows the spatial location of the addressee) are used in these examples. This suggests that on-line players can establish a sense of street players' position and activities using the reported positions confidently enough to be able to formulate directions and instructions in such terms.

The second category is messages where the street player appears to have a good idea of where the online player might be, but is less confident, for example questioning whether the street player is at a specific location. There are 112 such messages. Typical examples are:

Are you near a piece of scaffolding?

My map shows you near the bridge. Are you?

Did you just pass some steps?

The third category is messages where the online player gives general directions or makes geographical references that do not necessarily assume precise knowledge of the street player's location (although they also don't raise any doubts about it either). Such messages are broadly neutral with respect to the validity of positional information. There are 569 such messages. Typical examples are:

Now you need to find the steps

Go to 12 waterloo place

Head towards steps by George statue

Head for the big building with a flag on top

Waterloo place is near uncle roys office

The fourth category is messages that cast doubt on the usefulness or validity of reported positions or that appear to question the behavior of the positioning system in some way. These messages reflect moments when the operation of the positioning system may have been noticeable or even problematic for the online players. There are only 32 such messages including:

I can't pin point you

You are jumping all over the place on my map

Wow you move fast

Hi rachel? you keep coming and going

Your locator shows you standing still in the park is it broken?

How did you get over there?

Confirm your location cos this thing is not updating

Our fifth category is requests for location updates. There are 222 messages in which online players are enquiring about the location of a street player. Just over half of these appear to make specific requests for location updates via the map interface. The others are more general queries of the form 'where are you?'. These messages do not appear to cast doubt on the veracity of the position updates, for example questioning their accuracy, plausibility or commenting on jitter or other strange behaviours, although they do imply that online players would like more frequent updates.

What emerges from these observations is that while online players appear to be concerned about the frequency of reported positions (often asking for updates), they hardly appear to notice inaccuracies or other problems, and instead seem to be com- 
fortably working with reported position, often in a very precise way. Of course, the online players experience is not solely based on the positioning system. They also have access to other contextual information including audio messages from the street players. However, it seems that in spite of its apparent inaccuracy, self-reported positioning works well in an integrated way with the online map, audio and within the general context of this particular game. This can be contrasted with previous reports of GPS-based games that mix street players and online players is a similar manner and where apparently smaller errors became noticeable to online players, were commented on and even exploited them as part of the game. To understand why this might be so, we now look at the street players' experience and in particular, how they generated position updates.

\section{How and when positions are reported}

It seems that for the practical purposes of playing the game, self-reported positions are adequate to the task. On-line players can develop an adequate sense of where street players are for meaningful, game-related interaction to take place between them. In their turn, it seems that street players commonly report their positions in relation to city features at moments designed to be most useful to on-line players. Our evidence for this derives from our observations of how street players use the map in relation to their unfolding exploration of the streets. Three behaviors stand out.

\section{Declaring at landmarks and junctions}

Street players would often declare themselves to be at landmarks or junctions even when they were some distance away from them (e.g., half way along a street). We identified six key landmarks that provided focal points for declarations, including the two major entrances to the park, a café in the park, a major crossroads, the Duke of York statue and a crossing over a major road. Of course, the use of landmarks in wayfinding and the development of spatial knowledge of an area is well documented [10]. Beyond this however, our analysis suggests that this strategy of declaring at well defined locations such as landmarks is intended to produce clearer feedback from Uncle Roy and online players and to minimize misunderstandings concerning location.

\section{Looking ahead and declaring prospectively}

We observed players naturally position the map so that they could see further ahead than behind. They may do this to prepare themselves for the next leg of the journey, planning ahead and deciding where to go before actually reaching the next major decision point. However, as the 'me' icon is always located at the centre of the map, looking ahead requires them to position it in front of their actual physical position. 
We also saw examples of players explicitly declaring themselves to be ahead of their actual position. Sometimes this involved declaring a short distance (up to ten meters) ahead as in the following example:

While J is approaching the bridge from the east, he positions the 'me' icon at the centre of the bridge and declares about 5 meters to the east of the north end of the bridge. He then walks to the middle of the bridge and stops to look at the handheld computer.

In this and other similar examples, players appear to be anticipating time delay. Declaring a few seconds ahead of themselves provides time for the system to respond with new information (there was a delay of approximately six seconds between declaring and receiving a clue in return) and maybe even for them to digest it before they reach the next decision point - a strategy that will avoid them waiting around. On other occasions players declared themselves to be a longer distance (up to sixty meters) ahead of their location:

Having found herself unexpectedly back at the end of Carlon House Terrace where she'd been 10 minutes earlier, J. looks visibly frustrated. After asking directions and receiving more messages, she decides to head West on Carlton House Terrace. Halfway up, she stops, positions the 'me' icon at the Duke of York statue sixty meters further up the street, declares, and then waits for a response.

Again, our analysis suggests that players were using this strategy to obtain feedback (e.g. clue information and online player messages) in advance of taking a key decision. On several occasions players appeared to be unconfident about their direction and may have been confirming their chosen route (if already walking) or investigating a possible route (if stopped) so that they would know sooner rather than later whether they were heading in the wrong direction. This avoids the wasted time and effort that results from setting off on the wrong route, an important strategy in a game that is played against the clock. It should also be noted that the time delay involved in getting a response from an online player would be of the order of twenty seconds as they would have to compose and enter a text message.

In subsequent email feedback one of the players that we followed confirmed this strategy of declaring in advance of their position so as to obtain clues ahead of time:

"One thing I also remember doing was quite the opposite, that is, reporting my position in advance before I got there to have quicker feedback of whether or not I was on the right track. Maybe through a desire to anticipate and plan ahead ..."

\section{Looking behind and declaring retrospectively}

We also see some street players declaring and looking behind their current position. Panning behind would often occur when a player did not manipulate the map for a while and so physically moved ahead of their last reported position. Several map manipulations might then be required to realign their virtual position with their physical position, effectively recreating their recent path on the map. This of course results from not having an automated positioning system. However we also saw cases where players deliberately panned behind from their current map position, revisiting a previous location and then explicitly declaring, as in the following:

$C$. walks up from the lake to the next junction, then turns right...after about 15 meters, he stops, pans the map to the junction he has just passed and declares there. 
In this case the player decides to declare at a landmark that they have already passed. One reason for declaring behind was to retrigger clues from Uncle Roy as these did not remain persistently visible on the interface. Street players also sometimes redeclared a past position for the benefit of online players who had missed it as shown by the following feedback from our previous street player:

“... being pressured by players to report my position, which I probably repeated just to be sure they got the updates."

In summary, street players adopt various strategies for manipulating the map and declaring their position that (in purely numerical terms) generate large positioning errors. However, these strategies make perfect sense in terms of their experience of the game and furthermore, as our previous analysis of text messages suggests, also make sense to online players as part of ongoing collaboration.

\section{Plausibility, timing and communication}

These observations of how online and street players experience self reported positioning raise implications for how we think of self-reported positioning errors.

\section{Plausible errors}

It seems that the positioning errors generated by street players (if it is even appropriate to think of them as errors as we discuss later on) are plausible ones that make sense to the online players and that do not 'jar' with their expectations. Further insight into this claim is given by figure 5 which plots the positions of all street players' reported declarations on the game map. Visual inspection of this image suggests that a large majority of explicitly declared positions involve plausible locations (defined to be the streets, open squares and parkland) rather than implausible ones (in the middle of buildings or in the lake). This is backed up by statistical analysis. Of the 5,309 declarations plotted there were only $39(0.7 \%)$ where some part of the 'me' icon did not overlap with a plausible location. More surprisingly, the same is broadly true of map manipulations where for the 18,610 that we analyzed there were only $345(1.8 \%)$ where the 'me' icon did not overlap a plausible location. In short, reported positions are credible, even if at first sight they appear to involve a large distance error. 


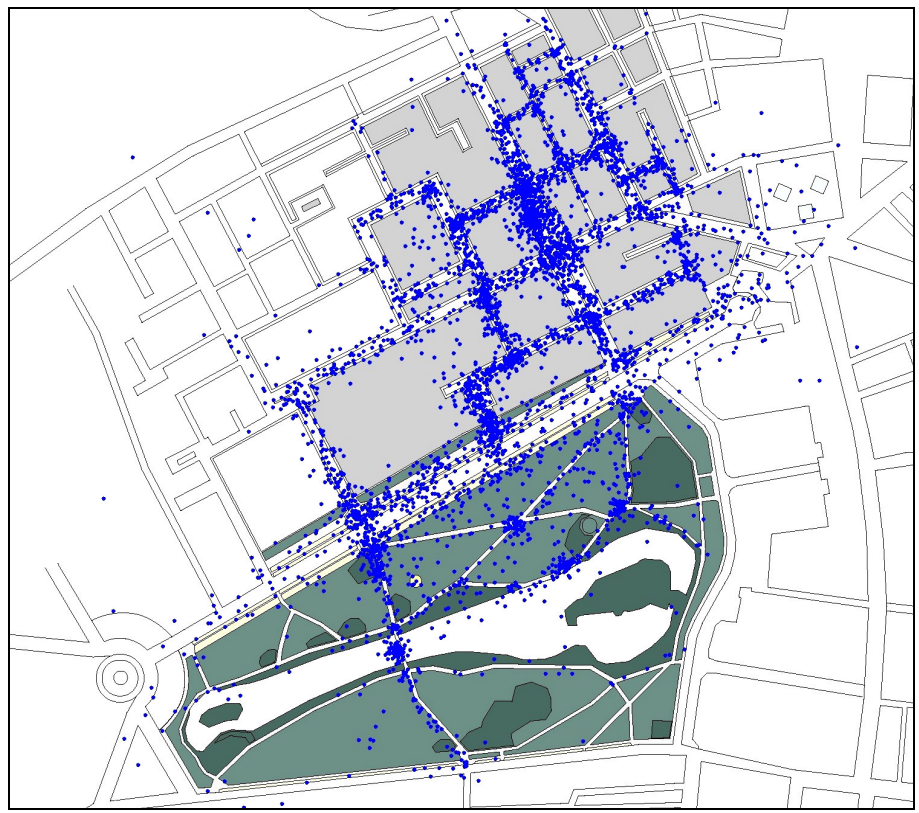

Figure 5: a plot of all explicitly declared positions

\section{Dislocation in time not space}

An alternative way to think of discrepancies between reported and actual position is in terms of time rather than space. Rather than reporting themselves to be at a different place, our street players are in fact reporting themselves to be at a different time. The strategies of declaring ahead and behind mean that reported positions tend to fall close to the player's actual physical path, one reason why they appear plausible. As noted previously, strategies such as declaring ahead are useful as they anticipate system delay and human response time. They also help to convey a sense of a player's trajectory through the streets. Indeed, a street player who always declared at the exact location they were at might seem sluggish to on-line players and might, in turn, have to stop and wait to receive relevant advice.

We can contrast this attention that people give to ensuring that positions are reported and received in ways which ensure the smooth flow of activity with automated positioning systems such as GPS which, due to the presence of network delays and system response time, effectively report a street player's position as it was several (in this case six or more) seconds ago. We speculate that in this particular game, GPS would fail to anticipate a player's requirement for information in advance of arriving at a decision point and even if available, might deliver information that was essentially out of date. 


\section{Reporting position as a communicative act}

We suggest that explicitly self-reported positions (declarations) should be interpreted as deliberate acts of communication, the intent of which is not so much to tell Uncle Roy and online players where the street player actually is at that very moment, but rather to solicit useful advice about a course of future action. In this context, declaring one's position is perhaps as much about deixis (pointing at and referencing features of the environment) as it is about telling someone exactly where you are. Put another way, self-reported position updates are not neutral pieces of information, but rather are imbued with meaning by a street player at the moment that they are generated. Again, this is something that is not captured by automated positioning systems such as GPS whose reported positions do not reflect any of the higher level semantics of the environment or the task at hand, or at least not at the point of data capture. With such systems, application-related semantics (e.g. what kind of 'context' the data are suggesting the application should be 'aware' of) has to be 'read in' after capture (e.g. by algorithms operating on the position data).

This observation reflects other studies of settings in which GPS data, though available, has not been used as anticipated. [8] describes an ambulance control room in which GPS was continually captured from ambulances but where many of the displays routinely used by controllers only updated positions at critical times in the emergency call (when an ambulance arrived at an incident, or at hospital). These junctures were notified by ambulance crews manually pressing keys on a small display in the ambulance cab. While alternative uses of GPS are certainly possible in control and similar domains, findings such as these are eminently understandable from the point of view of our study. Position data becomes relevant when it is timely in its delivery and, as with our players, is understood in terms of the unfolding trajectory of a journey and when communication between remote personnel is required.

\section{Revisiting the notion of error}

We began our analysis with a strictly numerical view of position error (computed as the difference between actual and reported location) as being typical of the way in which technology developers evaluate the performance of positioning systems. It now appears that a more subtle approach is required. Differences between reported and actual position that at first sight appear to be 'errors' may in fact naturally arise from appropriate strategies in which players communicate intent and accommodate delay while attending to the plausibility of their declared positions. As such, it may be inappropriate to think of them as being errors at all. Indeed, it may even be the case that, at least in these kinds of collaborative situations, automated positioning systems that superficially appear to be more accurate can in fact generate information with a timeliness not appropriate to the trajectory of ongoing user activities or to the specific requirements of communication between users. From this perspective data quality even at such an apparently 'low' level as raw position data - should be evaluated in 
terms of its appropriateness to its use-purpose and not just according to an abstract notion of error.

\section{Conclusions and broader issues}

Through enabling users to self-report their locations, we have presented a low-tech yet adequately reliable alternative to the automated capture of position data for use in the game 'Uncle Roy All Around You'. We have seen that players are able to navigate themselves through a city with help from others who see their location through selfreports. For the practical purposes of collaborative gaming, the declaration and map manipulation system we have designed does not introduce a great overhead - implicit reports fall out naturally from map manipulation and explicit declarations are easy to perform and motivated by the game's 'cover story'. In their turn, online players are able to work with self-reported positioning without greatly noticing inaccuracies.

However, the broader applicability of this approach is an open issue. Two potential limitations of self-reported positioning are that the mobile player has to know where they are and/or where they are heading, and that they may cheat, that is deliberately choose to lie about their position. The former limitation is clear - this is not an approach that will tell you where you are if you are lost. Rather, it is useful for applications where you are trying to inform other remote participants about your activities, especially where you are going. Potential uses are in remote guidance, command and control, arranging to meet or keeping others up to date with a background awareness of your general whereabouts - all activities that can occur outside of games. Cheating is clearly a possibility and self-reported positioning is not appropriate to situations in which users would be motivated to lie about their location and where this would cause a problem. However, in many situations users may not be motivated to lie and in others it may not be a problem (one can imagine games that involve remote users trying to work out where mobile users actually are based upon contextual cues).

As we noted in the introduction, one possible role for self-reported positioning is as a supplement to automated positioning systems, enabling the user to correct erroneous readings, fill in with self-reports while automated systems are unavailable, or possibly even take over from an automated system in order to disguise their position for a while to protect their privacy (another potentially useful reason for 'cheating').

A further issue for self-reported positioning is that it demands the constant engagement of the user in order to maintain an up to date position, and even then remote users may be frustrated at the low frequencies of updates. While this may be acceptable for tasks that are highly fore-grounded - such as playing an absorbing game - it may be less suited to more background tasks, for example where a context aware system spontaneously interrupts the user. We note an interesting tradeoff here between our experience of self-reported positioning and our previous experience with GPS. With the former, players have to continually work the technology to produce position updates, whereas the latter produces them automatically when it is available, but requires players to explicitly work the technology to maintain a fix and is unusable and arguably more visible (as a 'broken' technology) when not available. 
Whether ultimately this is a problem however, remains to be seen as it is still an open question as to what extent technologies that are ubiquitous should also fade into the background and become invisible. While this may seem an appealing idea, it raises serious challenges in terms of how users are expected to interact with invisible systems, see for example Bellotti et al's five questions for the designers of sensing based systems [2], and also raises the issue of whether users will ultimately accept technologies that monitor them continuously even when not being explicitly used.

Considering more immediate issues, one approach that we have adopted to try to deal with online players' frustration with infrequent updates is to change the representation of street players in the virtual world. In the most recent versions of 'Uncle Roy All Around You' (staged in Manchester and West Bromwich in the UK in May and June 2004) street players were shown as an avatar that walks an interpolated path between its current position and a newly reported position, giving the impression of continual movement and avoiding sudden jumps in apparent position. Our initial impression is that this refinement offers a much improved online representation.

A final issue concerning the future applicability of this work is whether automated positioning systems will improve to the point where self-reported positioning is no longer required as a low-tech fallback. It seems likely that automated approaches will continue to improve and this paper is not meant to be an argument against using them (we ourselves continue to work with GPS and other sensing systems in a variety of applications).

However, several points need to be borne in mind. First, actual large-scale user experiences reported to date (as opposed to demonstrations or controlled tests) suggest that designers should be careful not to underestimate how difficult it is to deploy technologies such as GPS in the wild and deliver a fluid and seamless experience. Second, improving the performance of sensing technologies may be as much a matter of economics as technical prowess. It may require a very large investment in additional sensors to achieve that last few percent of coverage. After all, why is it that even with a technology as widely used and well developed as GSM, we still routinely encounter communication blackspots when using standard mobile phones? Finally, we reiterate that even when they work, automated systems may not be providing the desired information. Studies of mechanisms such as self-reported positioning can identify new requirements for automated approaches, for example the need to reflect user intent.

Our work, then, opens out a new research challenge: how can we better integrate positioning systems with the natural ways in which humans orient to and communicate their location? Context aware systems need to develop a sense of context that truly is relevant to the activities that their users are performing. When this process is based on position data, exploiting natural features of human activity and communication at the point of data capture may provide solutions which help us to meaningfully measure the error of our ways. 


\section{Acknowledgements}

We gratefully acknowledge the support of the Engineering and Physical Sciences Research Council (EPSRC) through the Equator project, the Arts and Humanities Research Board (AHRB), the Arts Council of England and Microsoft.

\section{References}

1. Abowd, G., Atkeson, C., Hong, J., Long, S., Kooper R., Pinkerton, M., Cyberguide: A mobile context-aware tour guide. Wireless Networks, 3, 421-423, 1997.

2. Bellotti, V., Back, M., Edwards, W. K., Grinter, R. E., Henderson, A. and Lopes, C, Making Sense of Sensing Systems: Five Questions for Designers and Researchers, Proc. CHI 2002, CHI Letters, 1 (1), 415-422, Minneapolis, USA, 20-25 April, 2002, ACM.

3. Benford, S., Anastasi, R, Flintham, M., Drozd, A., Crabtree, A., Greenhalgh, C., Tandavanitj, N., Adams, M., Row-Farr, J., Coping with uncertainty in a location-based game, IEEE Pervasive Computing, September 2003, 34-41, IEEE.

4. Bjork, S., Falk, J., Hansson, R., Ljungstrand, P. (2001). "Pirates! Using the Physical World as a Game Board", Proc. Interact 2001, 2001, IFIP.

5. Brown, P. \& Jones, G., Context aware retrieval. Personal and Ubiquitous Computing, 5, 253-263, 2001

6. Flintham, M, Anastasi, R, Benford, S D, Hemmings, T, Crabtree, A, Greenhalgh, C M, Rodden, T A, Tandavanitj, N, Adams, M, Row-Farr, J, Where on-line meets on-the-streets: experiences with mobile mixed reality games, Proc. CHI 2003, Fort Lauderdale, Florida, 510 April 2003.

7. Imielinski, T., and Navas, J., GPS-based geographic addressing, routing, and resource discovery. Comm. ACM, 42 (4), 86-92, 1999.

8. Martin, D., Bowers J., Wastell, D., The interactional affordances of technology: An ethnography of human-computer interaction in an ambulance control centre. Proc HCI 1997: 263281, BCS.

9. Newman, W., Eldridge, M., \& Lamming, M., PEPYS: Generating autobiographies by automatic tracking. Proc. ECSCW 91, 1991, Kluwer.

10. Siegel, A. W., \& White, S. H., The development of spatial representations of large-scale environments. Advances in Child Development and Behavior, 10, 9-55, 1975.

11. Starner, T., Leibe, B., Singletary, B., \& Pair, J (2000b), "MIND-WARPING: Towards Creating a Compelling Collaborative Augmented Reality Game", Intelligent User Interfaces 2000, 256-259.

12. W. Piekarski and B. Thomas, "ARQuake: The Outdoors Augmented Reality System," Comm. ACM, vol. 45, no. 1, Jan. 2002, pp.36-38, ACM.

13. Want, R., Hopper, A., Falcao, V, \& Gibbons, J., The active badge location system. (1992) ACM Trans Information Systems, 10 (1), 91-102.

14. Want, R., Schilit, B., Adams, N., Gold, R., Petersen, K., Ellis, J., Goldberg, D., \& Weiser, M., The PARCTab ubiquitous computing experiment. Tech. report CSL-95-1, Xerox PARC, 1995. 